\section{Abstract PS2-06 \\ Comorbidities Associated With Onset of Atrial Flutter and Atrial Fibrillation in General Population}

Ravi K. Mareedu, MD, Marshfield Clinic; Ihab B. Abdalrahman, MD, Marshfield Clinic; Kodlipet C. C. Dharmashankar, MD, Marshfield Clinic; Robert T. Greenlee, PhD, Marshfield Clinic Research Foundation; Po-Huang Chyou, PhD, Marshfield Clinic Research Foundation; Sharma P. Param, MD, Marshfield Clinic; John J. Hayes, MD, Marshfield Clinic; Peter N. Smith, MD, Marshfield Clinic; Humberto J. Vidaillet, MD, Marshfield Clinic Research Foundation

Background: Atrial flutter (AFL) and atrial fibrillation (AF) are the most common atrial tachyarrhythmias. The conditions leading to the onset of one of these arrhythmias over the other remain largely unknown. Methods: To determine and compare the prevalence of known risk factors for cardiovascular disease among unselected individuals presenting with their first ever episode of AFL or AF, we evaluated 11 pre-selected clinical variables including age, sex, stroke, myocardial infarction, congestive heart failure (CHF), hypertension, rheumatic heart disease, smoking, chronic obstructive pulmonary disease (COPD), diabetes and thyroid abnormality. Using the resources of the Marshfield Epidemiologic Study Area, a database that captures nearly all medical care among its nearly 60,000 residents, all newly diagnosed cases of either AFL or AF in the region during a 4-year period were identified. Fischer's exact test was performed to compare the difference between AFL patients and AF patients for each of the 11 above-mentioned clinical characteristics. Multivariate logistic regression analysis was performed to obtain odds ratio (OR) and corresponding 95\% confidence interval (CI) for the status of AFL for each preexisting comorbidity, with adjustment for age and gender. Results: Among the 472 incident cases, 76 (16.1\%) had AFL and 396 (83.9\%) had AF. Compared to those with AF, subjects with AFL were more likely to have had a history of COPD (25\% vs. $12 \%, P=0.006)$ and $\mathrm{CHF}(28 \%$ vs. $17 \%, P=0.05)$. Hypertension on the other hand, was more common among individuals with AF (63\% vs. $47 \%, P=0.01)$. After adjusting for age and gender, patients with AFL as opposed to those with AF had higher odds for having a history of COPD (OR 2.34, 95\% CI 1.26-4.32, $P=0.007$ ), CHF (OR 2.00, 95\% CI 1.12-3.58, $P=0.019$ ), and lower odds of hypertension (OR $0.56,95 \% \mathrm{CI}$ $0.34-0.93, P=0.025)$. These statistically significant associations persisted even with COPD, CHF, and hypertension simultaneously included in a multivariate logistic regression model, in addition to age and gender. Conclusions: This study represents the first report to evaluate potential differences in the conditions associated with the development of AFL versus $\mathrm{AF}$ in man. This study raises an important question regarding the role of COPD and hypertension playing a role in initiation and maintenance of AFL and AF, respectively by their selective influence on the right atrial and left atrial dynamics, respectively.

\section{Abstract PS2-08}

\section{Hormone Therapy and Blood Pressure in an Elderly Cohort of Women}

Kristi Reynolds, PhD, MPH, Kaiser Permanente Southern California; Vicki Chiu, MS, Kaiser Permanente Southern California; Valerie Crooks, DSW, Kaiser Permanente Southern California

Background: Data on the effect of hormone therapy (HT) on blood pressure are limited and contradictory. Some clinical trials and epidemiologic studies have reported no net effect on blood pressure or decreases in blood pressure, while others have found that HT use increases systolic blood pressure. The objective of this study was to examine the association between HT use and blood pressure among an elderly cohort of women. Methods: Medical records were retrospectively reviewed from 1989 to 1999 among a subsample of women participating in a longitudinal study conducted at Kaiser Permanente Southern California. For each year, up to three blood pressure measures were abstracted randomly from among all blood pressure measurements recorded in the medical record. A total of 1021 postmenopausal women who had at least one blood pressure measurement at both baseline and follow-up were included in this analysis. Changes in blood pressure over time between HT users and nonusers were examined. Results: HT users $(\mathrm{n}=487)$ and nonusers $(\mathrm{n}=534)$ were similar in age at baseline with an overall mean age of $70.3 \pm 3.9$ years. The average duration of HT use was $27.6 \pm 12.0$ years. The mean duration of follow-up was $8.8 \pm 0.5$ years in HT users and
$8.7 \pm 0.6$ years in nonusers. Systolic and diastolic blood pressures were significantly higher at baseline among HT nonusers compared with users $(138.8 \pm 15.5 \mathrm{mmHg}$ vs. $135.9 \pm 16.1 \mathrm{mmHg}, P<0.01$; and $79.5 \pm 7.8 \mathrm{mmHg}$ vs. $77.7 \pm 7.9 \mathrm{mmHg}, P<0.001$, respectively). Systolic blood pressure increased over time among both HT nonusers and users, while diastolic blood pressure decreased over time. The average change in systolic blood pressure over time was significantly higher in HT users than nonusers $(7.0 \pm 0.9$ $\mathrm{mmHg}$ vs. $4.3 \pm 0.9 \mathrm{mmHg}$, respectively; $P=0.03$ ) and was independent of education, physical activity, cigarette smoking, alcohol consumption, and body mass index. The change in diastolic blood pressure was not statistically significant. Conclusion: These data suggest that elderly women who use HT have a larger increase in systolic blood pressure over time than nonusers; however, HT use does not affect diastolic blood pressure over time.

\section{Abstract PS2-09 \\ Racial Disparities in Actions to Control Blood Pressure in a Managed Care Organization}

Douglas W. Roblin, PhD, The Center for Health Research/Southeast, Kaiser Permanente Georgia; Junling Ren, MEd, The Center for Health Research/ Southeast, Kaiser Permanente Georgia; Edmund R. Becker, PhD, Rollins School of Public Health, Emory University

Background: The Seventh Report of the Joint National Committee on Prevention, Detection, Evaluation, and Treatment of High Blood Pressure (JNC VII) recommends lifestyle modifications (such as reducing sodium intake) to help control blood pressure (BP) in adults with hypertension. We analyzed variation by race in self-reported actions to control BP, both lifestyle activities and receipt of physician advice, within a random sample of adults with hypertension in a group-model managed care organization. Methods: We randomly sampled 300018 - to 74-year-olds with hypertension (750 from each of the 4 JNC VII hypertension stages) who had BP measured on at least 2 recent primary care visits. The survey, administered by telephone in 2007, included the Behavioral Risk Factor Surveillance System (BRFSS) Actions to Control Blood Pressure items and questions on race/ethnicity and socioeconomic status. Data on enrollee demographics, body mass index, antihypertensive medication use, and preexisting diabetes, hyperlipidemia, coronary artery disease and congestive heart failure were obtained from computerized administrative data. Lifestyle activities and receipt of physician advice were analyzed between black and white patients using descriptive and multivariate logistic regression analyses, adjusting for age and gender, BP control, obesity and pre-existing chronic conditions. Results: Seven hundred thirty-nine adults responded (25\% overall response rate, $58 \%$ cooperation rate - similar to the 2006 national BRFSS rates). Blacks ( $54 \%$ of respondents) were older, had worse BP control, and had higher prevalence of obesity and diabetes than whites (all $P=<0.05$ ). Blacks were more likely than whites to report changing dietary habits to control $\mathrm{BP}(88 \%$ vs. $82 \%, P=0.02)$ and to report receipt of advice on dietary habits, salt reduction, alcohol consumption, and exercise (all $P=<0.01$ ). The higher likelihood of blacks reporting receipt of advice remained significant $(\mathrm{OR}=1.6$ on alcohol consumption to $\mathrm{OR}=2.5$ on salt reduction, all $P=<0.01$ ) in multivariate models. Conclusions: In this sample of adults with hypertension, blacks were more likely than whites to report receipt of advice from physicians on actions to control BP. Although blacks had worse BP control and a higher prevalence of obesity, which had independent associations with receipt of advice, these factors did not account for the racial disparities. Further research is needed to clarify whether the racial differences in self-reported receipt of advice represent a recall or response bias, or other unmeasured patient or delivery system factors that affect likelihood of a patient receiving advice on actions to control BP. 